\title{
Validez en el registro del pico espiratorio máximo de niños asmáticos
}

\author{
de la Ciudad de México
}

\author{
Diana Jiménez-Millán, MSc, (I) Matiana Ramírez-Aguilar, M, MD, MSc, DSc, ${ }^{(1)}$ Hortensia Moreno-Macías, MSc, ${ }^{(1,2)}$ \\ Albino Barraza-Villarreal,MSc, (I) Blanca Estela del Río-Navarro, MD, (2) Isabelle Romieu, MD, MPH, ScD. ${ }^{(I)}$
}

\begin{abstract}
Jiménez-Millán D, Ramírez-Aguilar M, Moreno-Macías H, Barraza-Villarreal A, Del Río-Navarro BE, Romieu I. Validez en el registro del pico espiratorio máximo de niños asmáticos de la Ciudad de México. Salud Publica Mex 2007;49:182-189.
\end{abstract}

\section{Resumen}

Objetivo. Determinar la concordancia entre el pico espiratorio forzado (PEF) registrado por padres de niños asmáticos y el PEF electrónico que es el almacenado en el AirWatch (PEFe). Material y métodos. Se obtuvo el PEF de 42 niños de 5 a I5 años de edad del Hospital Infantil de México Federico Gómez de la Ciudad de México entre octubre de 1998 y 1999. Los padres registraron el valor máximo del PEF en un diario de salud. Se calculó la correlación de Spearman entre el PEFe y el PEFr. Utilizamos un modelo logístico de efectos mixtos. Resultados. La correlación del PEFe y el PEFr fue $r=$ $0.96(p<0.05)$ en niños con diagnóstico de asma moderada o severa y $r=0.40(p<0.05)$ en niños con asma leve. El tiempo de seguimiento y severidad del asma, el género y edad del niño y sus interacciones eran predictores de las diferencias entre el PEFe y el PEFr. Conclusiones. Los padres de niños de 6 a 8 años con asma moderada o severa reportan mejor el PEF en el diario de salud a lo largo del seguimiento que los padres de otros grupos.

Palabras clave: asma infantil; pico espiratorio máximo; diario de salud; México
Jiménez-Millán D, Ramírez-Aguilar M, Moreno-Macías H, Barraza-Villarreal A, Del Río-Navarro BE, Romieu I. Validity of peak flow record in asthmatic children residing in Mexico City.

Salud Publica Mex 2007;49:182-189.

\section{Abstract}

Objective. To determine the concordance between maximum peak expiratory flow records (PEFr) reported by the parents of asthmatic children and the electronic values stored by the AirWatch device (PEFe). Material and Methods. Records of PEF measurements between October 1998 and 1999 were obtained from 42 asthmatic children 5 to I 5 years of age recruited at the Hospital Infantil de Mexico Federico Gomez, in Mexico City. Parents recorded the maximum value in the health diary. Spearman correlation was calculated between PEFe and PEFr and a mixed-effects logistic model was used. Results. The correlation between PEFe and PEFr was $r=0.96$ $(p<0.05)$ among children with a diagnosis of moderate or severe asthma and $r=0.40(p<0.05)$ among children diagnosed with mild asthma. Follow-up time, asthma severity, gender and age of the child and their interactions were predictors of the differences between PEFe and PEFr. Conclusions. Parents of children with moderate or severe asthma from 6 to 8 years of age report PEF values with greater accuracy during follow-up than others.

Keywords: childhood asthma; peak expiratory flow; home monitoring; Mexico

(I) Instituto Nacional de Salud Pública. Cuernavaca, Morelos, México.

(2) Universidad Autónoma Metropolitana. México, DF.

(3) Hospital Infantil de México, Federico Gómez. México, DF.

Fecha de recibido: 2 I de octubre de 2006 - Fecha de aprobado: 24 de enero de 2007 Solicitud de sobretiros: Dra. Isabelle Romieu.Av. Universidad 655, col. Santa María Ahuacatitlán. 62508 Cuernavaca, Morelos, México. Correo electrónico: iromieu@correo.insp.mx 
E asma es un padecimiento que afecta significativamente la calidad de vida de los pacientes, pues en algunos casos llega a conducir a la incapacidad física, lo cual impacta tanto en la vida personal como en la dinámica familiar. ${ }^{1}$ En el caso donde los pacientes son niños, es necesario involucrar a los padres en el reconocimiento de los síntomas o cambios en la función pulmonar que pueden desencadenar en una crisis asmática. ${ }^{1}$

La vigilancia diaria de la presencia de síntomas y el monitoreo diario del pico espiratorio forzado (PEF, peack flow) son instrumentos de uso común en el manejo y control del asma y en las investigaciones clínicas y epidemiológicas, $^{2,3}$ lo que es recomendado por la GINA 2000 (The Global Initiatives for Asthma), que es una guía para el diagnóstico y tratamiento del asma. ${ }^{4}$ Dado que los padres de niños asmáticos generalmente están concientes de los problemas de salud de sus hijos, se esperaría que la información que proporcionansea verídicaen relación con la correcta lectura y reporte del PEF obtenido de equipos analógicos y actualmente de equipos electrónicos. ${ }^{5,6}$

Kamps A. y colaboradores compararon el registro del PEF escrito por padres de niños asmáticos y los datos del PEF almacenados en la memoria electrónica del flujómetro. ${ }^{7} \mathrm{El}$ porcentaje de datos correctos disminuyó de 56 a menos de $50 \%$ de la primera a la cuarta semana de seguimiento $(p<0.04)$. Por otro lado, estudios previos sobre la exactitud del PEF reportado por los padres usando flujómetros digitales señalan las discrepancias entre la fecha y veces en que se realizan las pruebas. ${ }^{7-10}$ Por lo antes mencionado, se planteó la pertinencia de realizar una investigación en la Ciudad de México en niños asmáticos que participaron en un estudio clínico aleatorizado, ${ }^{11}$ para determinar el grado de confianza del registro del PEF y que consecuentemente también se consideren con mayor confiabilidad las respuestas que dieron los padres a los apartados adicionales que contiene el diario de salud.

\section{Material y métodos}

Los datos del registro de los padres del PEF y los datos del PEF almacenados por el equipo electrónico forman parte de un estudio original (ensayo clínico aleatorizado) en el que se evaluó el efecto protector de la suplementación con antioxidantes en niños asmáticos de la Ciudad de México, quienes están expuestos de manera crónica a altos niveles de contaminantes foto-oxidantes. ${ }^{11}$

El estudio fue aprobado por los comités de Ética e Investigación del Instituto Nacional de Salud Pública y también se contó con el permiso de los padres de los niños participantes en el estudio por medio de la firma del consentimiento informado.
Del total de 158 niños que participaron con el manejo del AirWatch (Airway Monitoring System, ENACT Health Management Systems, Inc., Mountain View, California, EUA) sólo 42 niños completaron 12 semanas de registro escrito y electrónico y se incluyen en el análisis del presente artículo.

A cada participante se le proporcionó un diario de salud, el cual incluyó información sobre: síntomas respiratorios presentados durante el día, uso de medicamentos, ausentismo escolar, asistencia al médico o a la sala de urgencia por algún padecimiento respiratorio, tiempo que pasaba en el exterior y registro diario del PEF en $1 /$ min. La información se registró dos veces todos los días. A los padres se les indicó cómo debían llenar el diario de síntomas durante el periodo de estudio y se les instruyó sobre el uso del flujómetro. Los participantes realizaban una visita semanal al servicio de alergia del hospital, donde el diario de salud era revisado por enfermeras a cargo del seguimiento, así como la forma de utilizar el flujómetro con el fin de verificar el adecuado uso del equipo electrónico y el registro del PEF en el diario. En caso de detectar algún problema en el registro del PEF o uso del AirWatch, la enfermera instruía nuevamente a los participantes y a sus padres para corregir el manejo y registro.

El flujómetro fue programado para almacenar el mejor valor de las pruebas y con el valor predicho del PEF de acuerdo a la talla y peso de cada participante.

Para realizar la prueba se le solicitó al paciente que inspirara al máximo y exhalara con fuerza. Se les recomendó a los padres que la prueba se debía realizar siempre en la misma posición, de preferencia permaneciendo de pie. En cada prueba debían registrar la medición más alta de tres esfuerzos en el diario de salud (PEF escrito).

Durante el tiempo de seguimiento, el AirWatch almacenó cada una de las pruebas realizadas por los participantes, y ni ellos ni sus padres sabían que el equipo tiene memoria electrónica y estaba programado para almacenar la medición más alta (PEF electrónico).

\section{Análisis estadístico}

Para descartar la presencia de un sesgo de selección se comparó el grupo de los 42 niños incluidos en este análisis con el grupo de los 116 niños restantes a través de las variables de género, edad, severidad de asma, escolaridad de la madre y del padre y la asignación del tratamiento (placebo o vitaminas antioxidantes).

Se compararon los registros del PEF electrónico matutino contra el vespertino mediante la correlación de Pearson y se obtuvo una $r=0.9(p<0.05)$; de manera 
adicional se observó la distribución gráfica de los datos y se encontró que tenían un comportamiento similar en los dos turnos, por lo que se decidió considerar para el análisis los registros matutinos.

La severidad de asma fue determinada clínicamente por los médicos del servicio de alergia del hospital infantil en acuerdo con las recomendaciones de GINA ${ }^{4}$ y fue clasificada en dos grupos: uno con los niños que tuvieron el diagnóstico de asma leve y leve persistente y el otro con aquellos que tuvieron diagnóstico entre moderado y severo.

Se calcularon los coeficientes de correlación entre el PEF electrónico y escrito estratificando por edad, género, severidad de asma y escolaridad de la madre y padre. Se generó una variable que expresa la diferencia entre el PEF registrado en el AirWatch y el PEF escrito por los padres. A partir de la diferencia se generó una variable dicotómica, a la cual se le asignó 0 si la diferencia entre el PEF electrónico y el PEF escrito era entre cero y $10 \%$ de variabilidad con relación al PEF promedio registrado por el AirWatch para cada niño y 1 si la diferencia entre el PEF electrónico y el PEF escrito era mayor a $10 \%{ }^{12}$

Dado que el estudio es de tipo longitudinal y la probabilidad de que el reporte escrito sea diferente al electrónico y que varíe de un niño a otro a lo largo del tiempo debido a las características individuales y factores no medidos, se utilizó un modelo de regresión logística de efectos mixtos. ${ }^{13}$ Este tipo de modelo tiene la ventaja de no requerir el mismo número de observaciones por individuo como en el caso del análisis de varianza multivariado (MANOVA). ${ }^{14-16}$

La probabilidad de que exista una diferencia entre el PEF electrónico y el PEF escrito en este estudio se interpretó también como la probabilidad de error en el registro del PEF por los padres en el diario de salud, ya que se refiere a la existencia de una diferencia entre los dos valores que en teoría no debería existir; asimismo se consideró como estándar de oro al PEF electrónico.

Se realizó un análisis bivariado con el modelo logístico de efectos mixtos considerando las variables fijas: la edad, el género, la severidad de asma, la escolaridad del padre y de la madre. La variable de agrupación que fue el binomio madre-niño y el tiempo de seguimiento (semana) se consideraron como variables aleatorias. Además se evaluó el efecto modificador de las variables.

Las variables confusoras y los posibles modificadores de efecto que resultaron estadísticamente significativos, en un modelo múltiple, con una $p<0.05$ y con plausibilidad biológica se consideraron en el modelo final.

Dado que la prevalencia de cometer un error al reportar el PEF no es inferior al 0.1, no es posible interpretar la $R M$ como $R R$, por lo que para facilitar la interpreta- ción de los resultados, se calcularon las probabilidades ajustadas y sus respectivos intervalos de confianza.

\section{Resultados}

En el cuadro I se presentan las características basales de los niños participantes. El 48\% tenía edades entre 6 y 8 años, 67\% fueron niños, y 55\% tenía diagnóstico de asma leve intermitente persistente. El 74\% de las madres y $79 \%$ de los padres de los niños tenían estudios nivel secundaria y preparatoria o nivel superior.

El cuadro II muestra los coeficientes de correlación de Spearman entre el PEF escrito y el PEF electrónico estratificado por severidad de asma, edad de los niños, género, escolaridad de la madre y escolaridad del padre a través de las semanas de seguimiento. Los coeficientes de correlación más cercanos a 1 se observaron en el diagnóstico de asma moderada o severa con valores que van desde $r=0.96(p<0.05)$ a $r=0.81(p<0.05)$. Los valores más bajos del coeficiente de correlación se observaron en la edad de los niños de 9 a 11 años con un valor mínimo

\section{Cuadro I \\ Características sociodemográficas \\ Y DE SALUD DE LA POBLACIÓN DE ESTUDIO. Ciudad de MÉxico, I998-I999}

Características

n

$\%$

Edad de los niños (años cumplidos)

\begin{tabular}{lrr}
$6-8$ & 20 & 48 \\
\hline $9-11$ & 14 & 33 \\
\hline $12-17$ & 8 & 19
\end{tabular}

Género

\begin{tabular}{lll} 
Masculino & 28 & 67 \\
\hline Femenino & 14 & 33
\end{tabular}

Diagnóstico de severidad de asma

Leve intermitente-persistente

Moderada-severa

$\begin{array}{ll}23 & 55 \\ 19 & 45\end{array}$

Escolaridad de la madre

\begin{tabular}{lll} 
Primaria & II & 26 \\
\hline Secundaria & 13 & 31 \\
\hline Preparatoria-nivel superior & 18 & 43
\end{tabular}

Escolaridad del padre

\begin{tabular}{lrr} 
Primaria & 9 & 21 \\
\hline Secundaria & 14 & 33 \\
\hline Preparatoria-nivel superior & 19 & 46
\end{tabular}




\section{Cuadro II}

\section{Coeficientes de correlación de Spearman entre las variables del Pef del AirWatch y el PeF de los Padres. Ciudad de MéXico, 1998-1999}

\begin{tabular}{|c|c|c|c|c|c|c|c|c|c|c|c|c|}
\hline \multirow[b]{2}{*}{ Categorías } & \multicolumn{12}{|c|}{ Semanas de seguimiento } \\
\hline & I & 2 & 3 & 4 & 5 & 6 & 7 & 8 & 9 & 10 & 11 & 12 \\
\hline \multicolumn{13}{|l|}{ Severidad de asma* } \\
\hline Leve & 0.72 & 0.70 & 0.65 & 0.62 & 0.73 & 0.40 & 0.61 & 0.67 & 0.68 & 0.64 & 0.64 & 0.67 \\
\hline Moderada-severa & 0.91 & 0.96 & 0.81 & 0.81 & 0.93 & 0.93 & 0.92 & 0.95 & 0.93 & 0.96 & 0.86 & 0.88 \\
\hline \multicolumn{13}{|l|}{ Edad de los niños* } \\
\hline 6-8 años & 0.49 & 0.76 & 0.73 & 0.85 & 0.95 & 0.81 & 0.91 & 0.98 & 0.94 & 0.96 & 0.96 & 0.88 \\
\hline 9-II años & 0.80 & 0.84 & 0.44 & 0.57 & 0.38 & $0.4 \mathrm{I}$ & 0.43 & 0.47 & 0.64 & 0.67 & 0.63 & 0.72 \\
\hline $12-17$ años & 0.82 & 0.63 & 0.59 & 0.67 & 0.70 & 0.51 & 0.60 & 0.64 & 0.63 & 0.58 & 0.60 & 0.90 \\
\hline \multicolumn{13}{|l|}{ Género* } \\
\hline Masculino & 0.68 & 0.76 & 0.70 & 0.75 & 0.80 & 0.69 & 0.76 & 0.83 & 0.91 & 0.95 & 0.83 & 0.84 \\
\hline Femenino & 0.88 & 0.78 & 0.73 & 0.76 & 0.79 & 0.76 & 0.82 & 0.84 & 0.74 & 0.72 & 0.72 & 0.76 \\
\hline \multicolumn{13}{|l|}{ Escolaridad de la madre* } \\
\hline Primaria & 0.78 & 0.77 & 0.49 & 0.56 & 0.88 & 0.82 & 0.83 & 0.87 & 0.91 & 0.95 & 0.87 & 0.90 \\
\hline Secundaria & 0.81 & 0.77 & 0.43 & 0.45 & 0.83 & 0.91 & 0.78 & 0.81 & 0.88 & 0.97 & 0.93 & 0.93 \\
\hline Preparatoria-nivel superior & 0.83 & 0.77 & 0.62 & 0.68 & 0.81 & 0.70 & 0.84 & 0.88 & 0.81 & 0.75 & 0.68 & 0.87 \\
\hline \multicolumn{13}{|l|}{ Escolaridad del padre* } \\
\hline Primaria & 0.75 & 0.76 & 0.50 & 0.57 & 0.76 & 0.58 & 0.68 & 0.77 & 0.85 & 0.89 & 0.76 & 0.78 \\
\hline Secundaria & 0.75 & 0.69 & 0.71 & 0.85 & 0.78 & 0.69 & 0.78 & 0.81 & 0.80 & 0.79 & 0.74 & 0.80 \\
\hline Preparatoria-nivel superior & 0.83 & 0.87 & 0.43 & 0.49 & 0.83 & 0.63 & 0.60 & 0.65 & 0.74 & 0.66 & 0.76 & 0.79 \\
\hline
\end{tabular}

$* p<0.05$ con la prueba de Spearman

de $r=0.38(p<0.05)$ y un valor máximo igual a $r=0.84$ $(p<0.05)$.

En el análisis bivariado se determinaron como predictores importantes de la diferencia del PEF electrónico y el PEF escrito a la variable severidad de asma, género y edad de los niños. Adicionalmente, a través del ajuste de un modelo logístico múltiple se encontró que el riesgo de tener una diferencia entre la variable PEF electrónico y escrito era mas bajo $(\leq 10 \%)$ en los asmáticos moderados y severos (en comparación con asma leve), de sexo femenino, y mas jóvenes (6-7 años). Sin embrago, notamos una interacción entre el tiempo de seguimiento y el género ( $R M=1.27,95 \%$ IC $1.15-1.40)$, la edad $(R M=1.04,95 \%$ IC 1.027-1.06) y la severidad del asma ( $R M=1.19$, 95\% IC 1.09-1.30) (cuadro III).

Las figuras $1,2 \mathrm{a}$ y $2 \mathrm{~b}$ muestran que el comportamiento por severidad de asma, género y grupo de edad no siguen un comportamiento paralelo a lo largo de las semanas de seguimiento, es decir, ilustran las interacciones que cada una de estas variables tienen con el tiempo.

En la figura 1 se observa que la probabilidad de equivocarse en el grupo de padres con hijos que tienen asma leve es mayor que para el grupo de padres con hijos que tienen asma severa durante las primeras semanas de seguimiento. Sin embargo, la probabilidad de equivocarse para los padres que tienen hijos con asma leve disminuye a través del tiempo, al contrario del grupo de padres con hijos que tienen asma moderada o severa. Después de la semana 6 no se encontraron diferencias significativas entre los grupos de padres en cuanto a cometer errores en el registro.

Por otro lado, en la figura 2(a) se observan las probabilidades ajustadas promedio a través del tiempo de seguimiento en interacción con género. Durante las primeras 2 semanas, la probabilidad es menor si tienen 
Cuadro III

\section{Modelo de Regresión logística de efectos MiXtos \\ DE LAS DIFERENCIAS ENTRE LA VARIABLE PEF \\ ELECTRÓNICO Y EL PEF ESCRITO* POR LOS PADRES \\ en el diario de Salud. Ciudad de México, I998-1999}

\begin{tabular}{lccc} 
Variables & $R M$ & \multicolumn{2}{c}{$I C 95 \%$} \\
Severidad de asma (asma leve) & 0.20 & 0.10 & 0.42 \\
\hline Género (masculino) & 0.26 & 0.12 & 0.42 \\
\hline Edad de los niños(años cumplidos) & 0.83 & 0.73 & 0.94 \\
\hline Semana de seguimiento & 0.57 & 0.45 & 0.71 \\
\hline Severidad de asma por semana & 1.18 & 1.08 & 1.29 \\
\hline Género por semana & 1.27 & 1.14 & 1.41 \\
\hline Edad por semana & 1.04 & 1.02 & 1.06
\end{tabular}

* Diferencias en PEF es categórica: diferencia $<=10 \%$ es 0 , diferencia $>10 \%$ es I

$\mathrm{N}=42$

IC $95 \%$ : Intervalo de confianza al $95 \%$

$\mathrm{R}^{2}=50.7$

RM: razón de momio

hijos varones, sin embargo, aumentan conforme el tiempo de tal manera que de la semana 9 a la 12, los padres de las niñas tienen menor probabilidad de equivocarse.

En la figura 2(b) se observa el efecto modificador de la edad sobre la variable tiempo, de tal manera que después de la semana 6 , los padres de niños pequeños (6 a 8 años) son los que se equivocan menos y los de hijos más grandes (12 a 17 años) tienen mayor probabilidad de equivocarse. Se observa que la probabilidad más baja en la primera semana de seguimiento es para el grupo de edad de 12 a 17 años con $p=0.23$, mientras que la más alta es para el grupo de 6 a 8 años con $p=0.41$. No obstante, no se observa una diferencia significativa en los tres grupos de edad. A partir de la semana 6, las probabilidades promedio aumentan para el grupo de edad de 9 a 11 años y para el de 12 a 17 años conforme al tiempo de seguimiento, aunque para el grupo de edad de 12 a 17 años aumentan en mayor proporción que para el grupo de 9 a 11 años y las diferencias entre los grupos son más evidentes. Mientras tanto, para el grupo de edad de 6 a 8 años la probabilidad promedio disminuye a través del tiempo, es decir, que se equivocan menos al registrar en el diario de salud con relación a los padres de hijos de mayor edad.

\section{Discusión}

De acuerdo con los resultados de este estudio, se encontraron coeficientes de correlación de Spearman más cercanos a 1 en el diagnóstico de asma moderada o severa con valores que van desde $r=0.81(p<0.05)$ a $r=0.96(p<0.05)$ entre el PEF electrónico y el PEF escrito a través de las semanas de seguimiento. Asimismo, las probabilidades más bajas de encontrar una diferencia entre el PEF electrónico y el PEF escrito se ubican en el

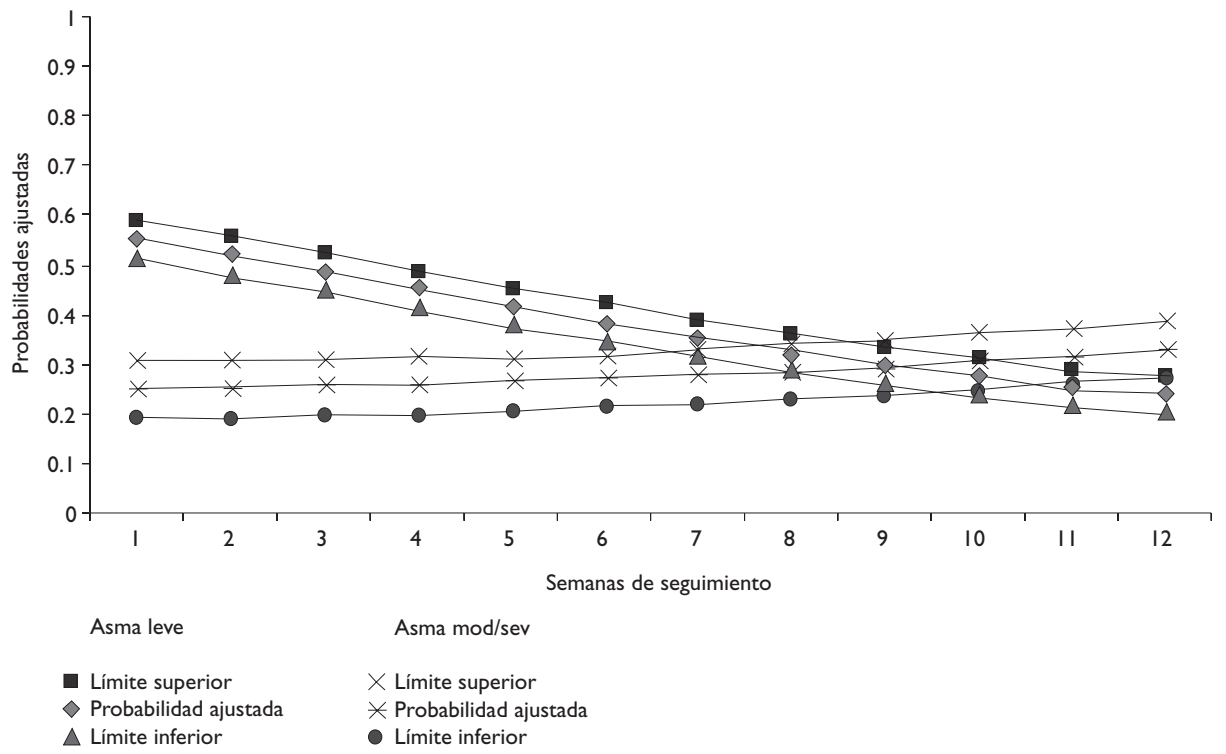

Figura I. Gráfica de las probabilidades de error en el registro del PEF por asma a través de las semanas de SEGUIMIENTO 

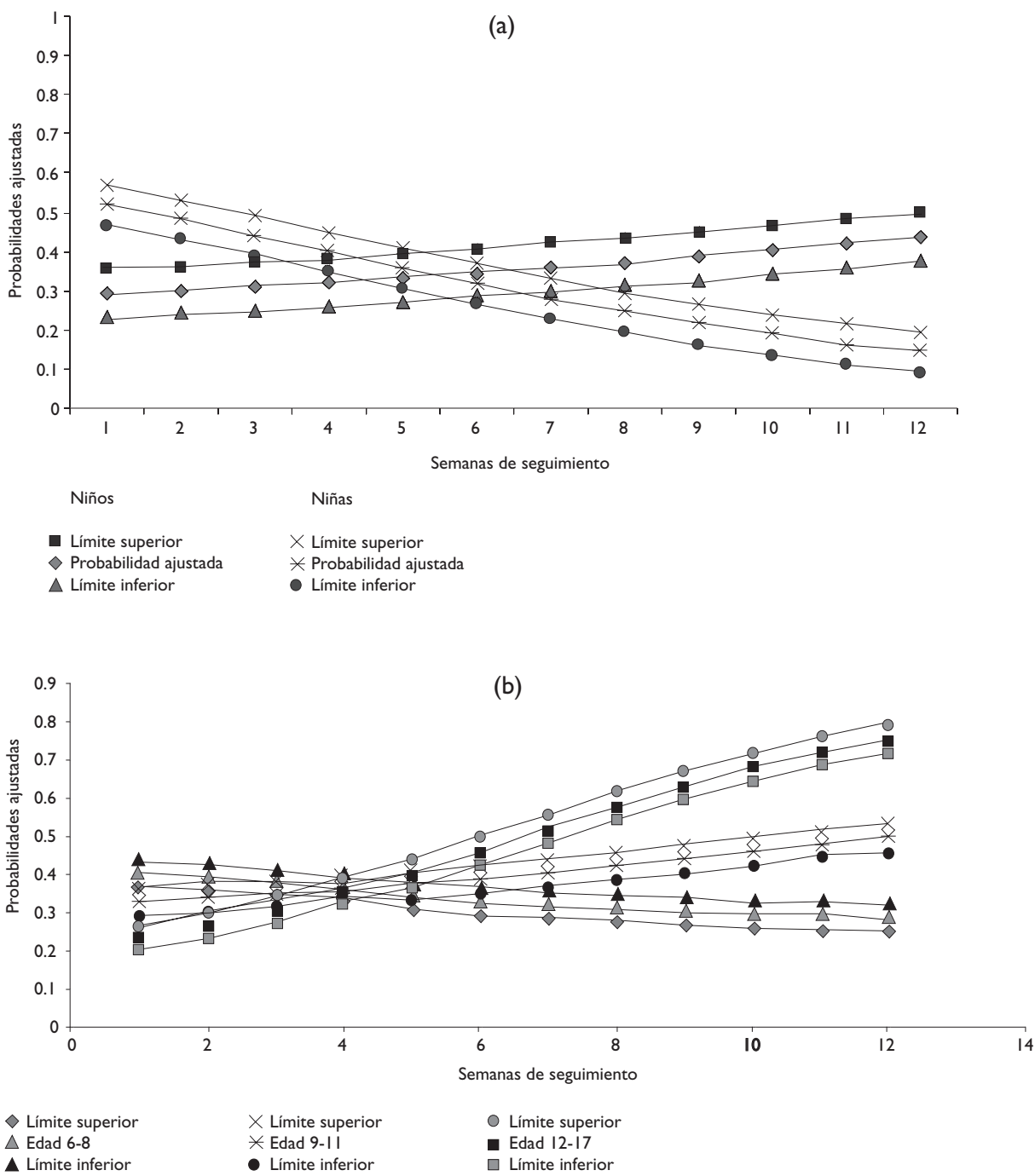

Figura 2. (a) Gráfica de las probabilidades de error en el Registro del PEF por género a través de las semanas de SEGUIMIENTO Y (B) GRÁFICA DE LAS PROBABILIDADES DE ERROR EN EL REGISTRO DEL PEF POR EDAD

grupo de padres que tienen hijos con asma moderada o severa; dichas probabilidades aumentan conforme al tiempo de seguimiento, pero el aumento no es mayor de 0.1 , es decir, que prácticamente se mantienen estables. Por otra parte, de acuerdo con el modelo, se observó que las variables predictoras de la diferencia entre el PEF electrónico y el PEF escrito son la severidad de asma, el género, la edad de los niños y las semanas de seguimiento, además de las interacciones severidad de asma por semana, género por semana y edad por semana.

Dentro de los resultados más importantes se destacan las probabilidades más altas encontradas en los grupos de edad de 9 a 11 años y de 12 a 17 años, las cuales aumentan conforme al tiempo de seguimiento; esto puede sugerir que los padres se equivocan más con hijos de mayor edad, lo que es similar a lo encontrado por Guyatt y colaboradores. ${ }^{17}$ Por otro lado y de manera similar, Lara y colaboradores ${ }^{18}$ encontraron diferencias significativas entre lo reportado por los padres y sus hijos mayores de 10 años.

Los resultados relacionados con el aumento de la probabilidad de error en el registro del PEF conforme al tiempo de seguimiento son consistentes con otros estudios $^{19-21}$ en los cuales se han encontrado discrepancias entre los registros de los padres y los datos almacenados en dispositivos electrónicos como el AirWatch, así como 
con la posible "distorsión" de los datos del diario, ${ }^{18}$ por lo que es probable que a lo largo del tiempo aumente la frecuencia de mediciones incorrectas más que el aprendizaje sobre el manejo adecuado del diario de salud. Wensley y colaboradores ${ }^{10}$ en su estudio sobre la calidad de las mediciones en el hogar de niños asmáticos señalan que el tiempo óptimo para recabar información fidedigna de mediciones diarias del PEF es de cuatro semanas de seguimiento. ${ }^{10}$

Este estudio sugiere que los padres de hijos diagnosticados con asma moderada y severa, a pesar del aumento de la probabilidad de error, presentan una mayor adherencia al registro correcto del PEF en el diario de salud que los padres de hijos diagnosticados con asma leve, lo que indica la posibilidad de que el primer grupo de padres sea más consciente de los riesgos de la enfermedad y por ello presten mayor atención al buen registro de los datos; esto último es consistente con el estudio de Kamps y colaboradores ${ }^{7}$ donde se determinó que los padres presentan una mejor adherencia al reporte de los datos cuando se les ha capacitado y sensibilizado acerca de la importancia del adecuado manejo, vigilancia y control de la enfermedad. ${ }^{7}$

Cabe señalar que el estudio puede considerarse innovador tanto por el hecho de identificar grupos de padres que pueden llegar a "distorsionar" los datos del PEF, como el género, la edad y el diagnóstico de severidad de asma, como por la determinación del efecto de estas variables sobre el registro del PEF, el cual no es independiente del tiempo de seguimiento. Estos resultados son similares a los reportados en una revisión sistemática realizada por Guevara y colaboradores ${ }^{22}$ donde señala que las intervenciones educativas para el manejo y control del asma en niños y adolescentes han sido más efectivas en los grupos de pacientes que tienen un grado moderado o severo de asma, pero no indican diferencias debidas al género o a la edad. ${ }^{22}$

Una limitación del presente estudio es la validez externa, ya que únicamente se puede extrapolar a poblaciones infantiles asmáticas de la Ciudad de México. Se considera que poblaciones de padres con hijos no asmáticos probablemente no son sensibles al problema de tener un hijo con una enfermedad crónica, por lo tanto la validez, en el caso del diario de salud, podría ser menor.

\section{Conclusiones}

Los resultados de este estudio sugieren una mayor adherencia a las instrucciones sobre cómo realizar el registro del diario de salud entre los padres que tienen niños con mayor grado de severidad de asma, por lo que resulta evidente que con una adecuada instrucción por parte del personal de salud sobre los riesgos y beneficios del correcto uso del diario de salud y del AirWatch a los padres, es posible disminuir la probabilidad de error en los grupos de padres que probablemente no estén lo suficientemente sensibilizados sobre el problema de asma de sus hijos, como lo son los padres de hijos con diagnóstico de asma leve. Basadas en datos de los autores de este trabajo las recomendaciones para quienes diseñan programas de seguimiento de niños asmáticos podrían ser: 1) cuando los estudios de seguimiento sean mayores a un mes, convendría establecer estrategias de recordatorio en el manejo y control del asma para los padres de niños con asma leve; 2) diseñar programas de capacitación para niños mayores de 10 años, ya que es probable (aunque no se confirmó en el estudio) que el control de su asma lo hayan hecho ellos mismos y no los padres.

La salud de niños asmáticos puede verse beneficiada por la adecuada vigilancia de la evolución del padecimiento, lo cual impactara tanto en la mejoría de la dinámica familiar como en los costos de atención médica que podrían disminuirse al identificar tempranamente signos de alarma en la evolución del padecimiento.

\section{Agradecimientos}

A la entusiasta participación de los niños y sus padres durante el presente estudio, así como a la Dra. Norma Isabel Reyes y a la Enf. Olegaria por todo su trabajo de supervisión y validación de la información durante el periodo de seguimiento del estudio. También a AirWatch por su apoyo para la obtención electrónica de los datos.

\section{Referencias}

I. Sierra-Monge JL, Del Rio-Navarro BE, Baeza -Bacab M.Asma. Salud Pública de México 1999;41:64-70.

2. Romieu I, Meneses F, Ruiz S, Huerta J, Sienra JJ,White M, et al. Effects of Intermittent Ozone Exposure on Peak Expiratory Flow and Respiratory Symptoms among Asthmatic Children in Mexico City.Arch Environ Health 1997; 52(5):368-376.

3. Hoek G, Wypij D, Brunekreef B. Self-reporting versus parental reporting of acute respiratory symptoms of children and their relation to pulmonary function and air pollution. Int J Epidemol 1999;28:293-299.

4. National Heart, Lung and Blood Institute. Global Strategy for Asthma Management and Prevention. [Consultado: Febrero 2006]. Disponible en: http://www.ginasthma.org.

5. Romieu I, Meneses F, Ruiz S, Sienra JJ, Huerta J,White M, et al. Effects of air pollution on the respiratory health of asthmatic children living in Mexico City.Am J Respir Crit Care Med 1996;154:300-307.

6. Doherty GB, Ross RN, Ross PE. The Effectiveness of An Interactive Electronic Lung Function Monitoring System in the Total Management of Refractory Asthma. Dis Manage Health Outcomes 1998:3(2):89-98. 
7. Kamps WA, Roorda RJ, Brand PLP. Peak Flow diaries in childhood asthma are unreliable. Thorax 200 I;56:180-182.

8. Laster J, Burdis-Jones S, Palmer J, Cochrane GM. How acute asthma diary cards? Thorax 1989;44:343.

9. Hyland ME, Kenyon CAP,Allen R, Howarth P. Diary keeping in asthma comparison of written and electronic methods. BMJ 1993;306:487-489.

10.Wensley DC, Silverman M. The quality of home spirometry in school children with asthma. Thorax 200 I;56: 183-185.

I I. Romieu I, Sierra-Monge J], Ramírez-Aguilar M,Téllez-Rojo MM, Moreno-Macías H, Reyes-Ruiz NI, et al. Antioxidant Supplementation and Lung Functions among Children with Asthma Exposed to High Levels of Air Pollutants.Am J Respir Crit Care Med 2002;166:703-709.

12. Boezen HM, Van der Zee SC, Postma DS, Vonk JM, Gerritsen J, Hoek G, et al. Effects of ambient air pollution on upper and lower respiratory symptoms and peak expiratory flow in children. Lancet 1999;353(9156):859-860

13. Zar JH. Bioestadistical Analysis. 3a. edición. New Jersey: Prentice Hall, 1996.

14. Cnann A, Laird NM, Slasor P. Using the General Linear Mixed Model to analyze unbalanced repeated measures and longitudinal data. Statist Med 1997; 16:2349-2380

15. Cannon MJ,Warner L, Taddei JA, Kleinbaum D.What can go wrong you assume that correlated data are independent: and illustration from the evaluation of childhood health intervention in Brazil. Statist Med 200I;20:146I-I 467.

16. Kung-Yee L, Zeger S. Longitudinal data analysis using generalized linear models. Biometrika 1986;17:13-22.

17. Guyatt GH, Juniper EF, Griffith LE, Feeny DH, Ferrie PJ. Children and adult perceptions of childhood asthma. Pediatrics 1997;99:165-168.

18. Lara M, Duan N, Sherbourne C, Lewis MA, Landon C, Halfon N, et al.

Differences between child and parent reports of symptoms among latino children with asthma. Pediatrics 1998;102:1-8.

19. Reddel HK, Toelle BG, Marks GB,Ware SI, Jenkins CR, Woolcock AJ. Analysis of adherence to peak flow monitoring when recording of data is electronic. BJM 2002;324: I46-I 47.

20.Verschelden P, Cartier A, L'Archevê J, Trudeau C, Malo JL. Compliance with and accuracy self-assessment of peak expiratory flow (PEF) in asthmatic subjects over a three month period. Eur Respir J 1996;9(5): 880-885.

21. Chowienczyk PJ, Parkin DH, Lawson CP, Cochrone GM. Do asthmatic patients correctly record home spirometry measurements? BJM 1994;309:1618.

22. Guevara JP, Wolf FM, Grum CM, Clark NM. Effects of educational interventions for self management of asthma in children and adolescents: systematic review and meta- analysis. BMJ 2003;326:I-6. 